\title{
S phase-dependent interaction with DNMT1 dictates the role of UHRF1 but not UHRF2 in DNA methylation maintenance
}

\author{
Jiqin Zhang ${ }^{1, *}$, Qinqin Gao ${ }^{1, *}$, Pishun $\mathrm{Li}^{1}$, Xiaoli Liu ${ }^{1}$, Yuanhui Jia ${ }^{1}$, Weicheng $\mathrm{Wu}^{1}$, Jiwen $\mathrm{Li}^{1}$, Shuo Dong ${ }^{2}$, \\ Haruhiko Koseki ${ }^{3}$, Jiemin Wong ${ }^{1}$ \\ ${ }^{I}$ The Institute of Biomedical Sciences and School of Life Sciences, East China Normal University, 500 Dongchuan Road, Shanghai \\ 200241, China; ${ }^{2}$ Department of Medicine, Baylor College of Medicine, Houston, TX77030, USA; ${ }^{3}$ Riken Research Center for Al- \\ lergy and Immunology, 1-7-22 Suehiro, Tsurumi-ku, Yokohama 230-0045, Japan
}

Recent studies demonstrate that UHRF1 is required for DNA methylation maintenance by targeting DNMT1 to DNA replication foci, presumably through its unique hemi-methylated DNA-binding activity and interaction with DNMT1. UHRF2, another member of the UHRF family proteins, is highly similar to UHRF1 in both sequence and structure, raising questions about its role in DNA methylation. In this study, we demonstrate that, like UHRF1, UHRF2 also binds preferentially to methylated histone $\mathrm{H3}$ lysine 9 (H3K9) through its conserved tudor domain and hemi-methylated DNA through the SET and Ring associated domain. Like UHRF1, UHRF2 is enriched in pericentric heterochromatin. The heterochromatin localization depends to large extent on its methylated H3K9-binding activity and to less extent on its methylated DNA-binding activity. Coimmunoprecipitation experiments demonstrate that both UHRF1 and UHRF2 interact with DNMT1, DNMT3a, DNMT3b and G9a. Despite all these conserved functions, we find that UHRF2 is not able to rescue the DNA methylation defect in Uhrf1 null mouse embryonic stem cells. This can be attributed to the inability for UHRF2 to recruit DNMT1 to replication foci during $S$ phase of the cell cycle. Indeed, we find that while UHRF1 interacts with DNMT1 in an S phase-dependent manner in cells, UHRF2 does not. Thus, our study demonstrates that UHRF2 and UHRF1 are not functionally redundant in DNA methylation maintenance and reveals the cell-cycle-dependent interaction between UHRF1 and DNMT1 as a key regulatory mechanism targeting DNMT1 for DNA methylation.

Keywords: UHRF2; UHRF1; DNA methylation; histone methylation; DNMT1

Cell Research (2011) 21:1723-1739. doi:10.1038/cr.2011.176; published online 8 November 2011

\section{Introduction}

DNA methylation is a prototype of epigenetic modification widely present in eukaryotes. In mammals, DNA methylation mainly occurs at cytosine-C5 in the context of $\mathrm{CpG}$ dinucleotides. This modification is essential for embryonic development and plays critical roles in transcriptional regulation, heterochromatin formation, X-inactivation, imprinting and genome stability [1]. Aberrant DNA methylation has been implicated in many human

\footnotetext{
*These two authors contributed equally to this work.

Correspondence: Jiemin Wong

E-mail: jmweng@bio.ecnu.edu.cn

Received 6 July 2011; revised 31 August 2011; accepted 6 September 2011; published online 8 November 2011
}

diseases including cancers [2].

The pattern of DNA methylation is believed to be established in early development by de novo DNA methyltransferases DNMT3a and DNMT3b, and then maintained primarily by the activity of DNMT1 [3]. DNMT1 has a strong preference for hemi-methylated $\mathrm{CpG}$ substrates generated during DNA replication [4], a property ideal for maintaining the stable inheritance of DNA methylation. Consistent with its role in DNA methylation maintenance, DNMT1 is recruited to DNA replication forks in S phase and co-localizes with pericentric heterochromatin foci that are replicated in middle and late $\mathrm{S}$ phase $[4,5]$. Although DNMT1 interacts with proliferating cell nuclear antigen, a cofactor of DNA polymerase delta, and this interaction was thought to target DNMT1 to replication forks [6], recent studies demonstrate that UHRF1, also known as ICBP90 and NP95, interacts with 
DNMT1 and is required for targeting DNMT1 to replication forks [7, 8]. This function of UHRF1 is conserved in evolution, as homologs of UHRF1 are implicated in DNA methylation in Arabidopsis and zebrafish [9, 10].

UHRF1 was initially identified independently as an inverted CCAAT Box-binding protein and a gene highly expressed in proliferating cells $[11,12]$. Structurally UHRF1 harbors at least five functional domains in the order from $\mathrm{N}$ - to $\mathrm{C}$-terminus: ubiquitin-like domain (UBL), a tandem tudor domain, a plant homeodomain (PHD), a SET and Ring associated (SRA) domain and a really interesting new gene (Ring) domain (see Figure 1A). A subsequent study showed that UHRF1 binds methylated $\mathrm{CpG}$ through its SRA domain and plays a role in repression of cell cycle inhibitors such as p21 [13]. However, the most striking discovery for UHRF1 is likely its function in DNA methylation. Much like Dnmt1 null embryonic stem (ES) cells, the Uhrfl null mouse ES cells are severely impaired in DNA methylation [7, 8]. Mechanistically, UHRF1 was found to bind hemi-methylated $\mathrm{CpG}$ through its SRA domain and this activity appears to be required for targeting DNMT1 to replication forks [14-16]. In addition to its hemi-methylated $\mathrm{CpG}$ binding activity, UHRF1 also binds preferentially to diand tri-methylated lysine 9 of histone $\mathrm{H} 3$ (H3K9me2/3) $[17,18]$. The PHD domain and tudor domain have been implicated in the binding of $\mathrm{H} 3 \mathrm{~K} 9 \mathrm{me} 2 / 3$ [17-19]. In agreement with the presence of a Ring domain, UHRF1 has an E3 ligase activity that ubiqutinates histones and non-histone proteins [17]. Given its ability to recognize both methylated DNA and methylated H3K9, UHRF1 not only plays a critical role in DNA methylation maintenance but may also mediate a cross-talk between DNA and histone methylation.

During evolution, the presence of UHRF1 seems to correlate well with the DNA methylation status of the organisms, as UHRF1 homolog is absent in yeast, $C$. elegans and Drosophia, organisms without substantial DNA methylation. While the zebrafish genome contains only one homolog of UHRF1, mouse and human genomes contain additionally a UHRF2 gene that encodes a protein highly homologous to UHRF1 in sequence and structure (Figure 1A). UHRF2, also known as NIRF, has been shown to be cell cycle-regulated and has autoubiquitination activity [20-22]. The substantial sequence and structural conservation between UHRF1 and UHRF2 argues for a potential functional conservation between these two proteins. As UHRF1 and UHRF2 are the only proteins in the human genome that contain an SRA domain, this raises the question as to whether UHRF2 also recognizes hemi-methylated DNA and plays a role in DNA methylation maintenance. In this study, we show that, like UHRF1, UHRF2 also binds preferentially to methylated histone $\mathrm{H} 3 \mathrm{~K} 9 \mathrm{me} 2 / 3$ through the conserved tudor domain and hemi-methylated DNA through the SRA domain, and interacts with DNMT1, DNMT3a, DNMT3b and G9a. Interestingly, we found that the SRA domain of UHRF2 exhibited a better binding activity for fully methylated DNA than the SRA domain of UHRF1. Importantly, we found that expression of UHRF2 cannot rescue the DNA methylation defect in Uhrf1 null mouse ES cells. Mechanistically we found that UHRF2 lacks an $\mathrm{S}$ phase-dependent interaction with DNMT1 that is characteristic of UHRF1.

\section{Results}

\section{UHRF2 recognizes specifically $\mathrm{H} 3 \mathrm{~K} 9 \mathrm{me} 2 / 3$}

Given the substantial sequence and structural homology between UHRF1 and UHRF2 (Figure 1A), we first attempted to test if UHRF2 also selectively binds H3K9me2/3. We first immobilized biotinylated synthetic $\mathrm{H} 3$ or $\mathrm{H} 4$ peptides with various methylation pattern to streptavidin agarose beads and incubated them with HeLa nuclear extracts. After washing, the peptide-bound proteins were resolved by SDS-PAGE and analyzed for the presence of UHRF2 by western blot. A representative result in Figure 1B shows that, like UHRF1, UHRF2 also bound to the $\mathrm{H} 3$ peptides containing K9me2/3. Similarly, western blot analysis revealed the specific binding of $\mathrm{K} 9 \mathrm{me} 2 / 3$ by HP $1 \alpha$, a prototypic $\mathrm{H} 3 \mathrm{~K} 9 \mathrm{me} 2 / 3$-binding protein $[23,24]$. As additional controls, western blot analyses showed that PHF 8 specifically bound to the H3 peptides with $\mathrm{H} 3 \mathrm{~K} 4 \mathrm{me} 2 / 3$, whereas HDAC1, a subunit of the NuRD complex, bound prominently to unmodified $\mathrm{H} 3$, but poorly to $\mathrm{H} 3 \mathrm{~K} 4 \mathrm{me} 1-3$, in full agreement with previous studies [25-28]. It is noteworthy that in comparison to UHRF1, we observed in multiple experiments a better binding of $\mathrm{H} 3 \mathrm{~K} 9 \mathrm{me} 3$ than $\mathrm{H} 3 \mathrm{~K} 9 \mathrm{me} 2$ by UHRF2. In addition, $\mathrm{H} 3 \mathrm{~K} 4 \mathrm{me} 2$ appears to inhibit the H3K9me2 binding by UHRF1, UHRF2 and HP1 $\alpha$, as these proteins bound poorly to the $\mathrm{H} 3$ peptide with dimethylations at both $\mathrm{K} 4$ and $\mathrm{K} 9$ residues. Altogether, these results demonstrate that both UHRF1 and UHRF2 in HeLa nuclear extracts specifically bind $\mathrm{H} 3 \mathrm{~K} 9 \mathrm{me} 2 / 3$.

\section{Tudor domain of UHRF2 is important for $\mathrm{H} 3 \mathrm{~K} 9 \mathrm{me} 2 / 3$ binding specificity \\ Previous studies on UHRF 1 have mapped its H3K9me2/3 binding activity to the PHD and tudor do- main [17-19]. Similarly, by using in vitro-translated and ${ }^{35} \mathrm{~S}$-methionine-labeled full-length and deletion mutants of UHRF2 and peptide pull-down assays, we found that the UHRF2 (aa 1-435) and UHRF2 (aa 119-397) frag-}


ments containing both the tudor and PHD domains were sufficient for binding H3K9me2/3 (Figure 1C). Furthermore, point mutations of two conserved hydrophobic and aromatic residues within the tudor domain (WF143/144 and FW262/263AA) abolished the H3K9me2/3-binding activity of UHRF2 (Figure 1C), indicating a critical role for the tudor domain in mediating the binding of H3K9me2/3. We note that both UHRF1 and UHRF2
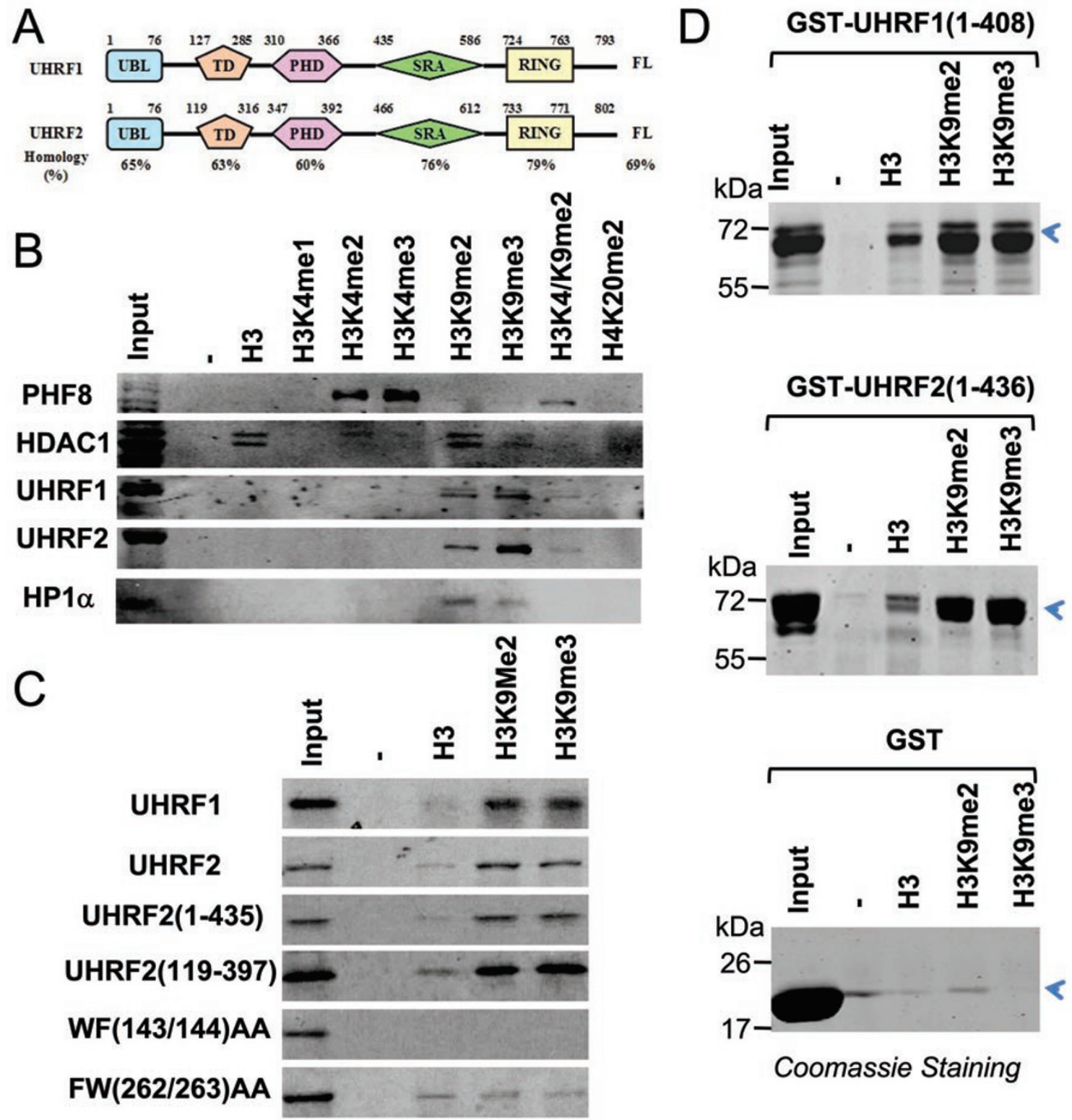

Figure 1 UHRF2 recognizes specifically H3K9 methylation by its tandem tudor domain. (A) A diagram illustrating the structure and sequence similarity between human UHRF1 and UHRF2. UBL, ubiquitin-like domain; TD, tandem tudor domain; PHD, plant homeodomain; SRA, SET and ring associated domain; Ring, really interesting new gene domain. (B) Binding of UHRF2 to a panel of methylated histone $\mathrm{H} 3$ and $\mathrm{H} 4$ peptides in comparison to other known histone-binding proteins. Pulldowns with HeLa nuclear extracts and various histone peptides were performed and the binding of UHRF1, UHRF2, HP1 $\alpha$, PHF8 and HDAC1 were revealed by western blot analysis. (C) The tandem tudor domain determines the H3K9me2 binding specificity. In vitro synthesized, ${ }^{35} \mathrm{~S}$-met-labeled UHRF2 and deletion or point mutation mutants were subjected to pull-down assays with $\mathrm{H} 3$ and $\mathrm{H} 3 \mathrm{~K} 9 \mathrm{me} 2$ and $\mathrm{H} 3 \mathrm{~K} 9 \mathrm{me} 3$ peptides. The binding of various UHRF2 proteins were revealed by autoradiography. (D) Recombinant UHRF1 and UHRF2 bound H3K9me2/3 peptides in vitro. Purified GST-UHRF1 (aa 1-436) and GSTUHRF2 (aa 1-408) were subjected to pull down assays and the proteins bound to the peptides were resolved by SDS-PAGE and revealed by Coomassie blue staining. 
exhibited a weak binding activity toward unmodified $\mathrm{H} 3$ peptide, in agreement with the recent reports that the PHD domain of UHRF1 can interact with unmodified H3 N-terminal tail [29-31].

To test if UHRF2 binds directly to the methylated histone $\mathrm{H} 3$, we expressed and purified UHRF2 aa 1-436 as a GST fusion protein from bacteria. Subsequent pull down followed by Coomassie blue staining showed that this protein bound preferentially to the $\mathrm{H} 3 \mathrm{~K} 9 \mathrm{me} 2 / 3$ peptides and weakly to the unmodified $\mathrm{H} 3$ peptide (Figure 1D). Similarly, the GST-UHRF1 (aa 1-408) fusion protein also exhibited the same binding specificity. As no binding was observed for control GST proteins, these results clearly demonstrate that binding of $\mathrm{H} 3 \mathrm{~K} 9 \mathrm{me} 2 / 3$ is intrinsic to both UHRF1 and UHRF2.

UHRF2 binds hemi-methylated DNA through the conserved SRA domain

The SRA domain in UHRF1 binds selectively to hemi-methylated DNA. As this domain is found only in UHRF1 and UHRF2, we next determined whether UHRF2 also binds hemi-methylated DNA via its SRA domain. We first expressed and purified recombinant UHRF2 containing either tudor, PHD and SRA (aa 78626 ) or the SRA domain alone (aa 416-626) in the form of GST-fusions from bacteria (Figure 2A). As a positive control, we also expressed and purified GST-UHRF1 aa 95-610 fragment (Figure 2A). The purified proteins were subjected to gel mobility shift assays using a ${ }^{32} \mathrm{P}$-labled oligonucleotide probe with or without hemi-methylated CpGs. Gel mobility shift assays in Figure 2B show that both UHRF1 (95-610) and UHRF2 (78-626) bound hemi-methylated but not unmethylated probe in a dosedependent manner. In addition, GST-UHRF2 (416-626), which contains only the SRA domain, bound the hemimethylated DNA probe as effectively as GST-UHRF2 (78-626) (Figure 2C), indicating that the SRA domain is sufficient for the binding of hemi-methylated DNA probe.

As UHRF1 was initially identified as a methyl-CpG binding protein, we next tested whether UHRF2 binds fully methylated DNA. In this regard, we compared the binding of GST-UHRF1 (95-610) and GST-UHRF2 (78$626)$ to the same probe that was either un-, hemi- or fully methylated. Interestingly, we found that while GSTUHRF1 (95-610) exhibited virtually no binding to fully methylated probe, GST-UHRF2 (78-626) bound weakly to the fully methylated probe compared to hemi-methylated probe (Figure 2D). This difference was verified in subsequent gel shift assays using fully methylated probes and an increasing amount of GST-UHRF1 (95-610) and GST-UHRF2 (78-626) proteins (Figure 2E). Finally, based on the sequence conservation between UHRF1 and UHRF2 SRA domains, we generated GST-UHRF2 (416626) with alanine mutation at R520 or Y584, respectively (Figure 2F). The R520 and Y584 in UHRF2 are equivalent to R491 and Y555 in UHRF1, both of which are critical for the binding of hemi-methylated DNA by the UHRF1 SRA domain $[14,15]$. As shown in Figure $2 \mathrm{G}$, these mutations impaired the hemi-methylated DNA-binding activity of UHRF2 SRA. In addition, these mutations also impaired the binding of UHRF2 SRA domain to fully methylated probe (Figure $2 \mathrm{H}$ ). Finally, we found that full length Flag-tagged UHRF1 and UHRF2 expressed and purified from 293T cells also bound hemimethylated but not unmethylated DNA probe (Supplementary information, Figure S1). Thus, much like the SRA domain in UHRF1, the SRA domain from UHRF2 also binds hemi-methylated DNA. Our results also revealed that the SRA domain of UHRF2 also exhibits a stronger binding activity toward fully methylated probe than that of UHRF1.

Constitutive pericentric heterochromatin localization of UHRF1 and UHRF 2 v middle and late $S$ phase pericentric heterochromatin localization of DNMT1

We and others have shown that in HeLa and NIH3T3 cells UHRF1 is enriched in pericentric heterochromatin $[17,32,33]$. To test the potential role of UHRF2 in targeting DNMT1 for DNA methylation, we next analyzed its subcellular localization in S phase in NIH3T3 cells. For this purpose, NIH3T3 cells were transfected with GFP-UHRF1 or GFP-UHRF2 for $36 \mathrm{~h}$ and then cultured with media containing BrdU for $4 \mathrm{~h}$ and processed for BrdU immunostaining. We scored cells with relatively diffuse BrdU staining or bright foci staining overlapped with DAPI staining as cells in the early or middle and late S phase, respectively. As shown in Figure 3, both UHRF1 and UHRF2 exhibited constitutive pericentric heterochromatin staining throughout the S phase. Consistent with previous data from mouse ES cells, DNMT1 was clearly enriched and colocalized with UHRF1 and UHRF2 in pericentric heterochromatin in cells in middle and late $\mathrm{S}$ phase. Similarly, we found that endogenous UHRF2 was enriched in approximately 70\% NIH3T3 cells in the pericentric heterochromatin regions that were observed as bright DAPI staining foci (Figure 4A) and exhibited a diffuse nuclear staining in $\sim 30 \%$ cells.

\section{Both H3K9me2/3 and hemi-methylated DNA binding ac- tivities contribute to UHRF2 pericentric heterochromatin localization \\ To investigate if methylated $\mathrm{H} 3 \mathrm{~K} 9$ and/or methylated DNA binding activity of UHRF2 contribute to its het-}




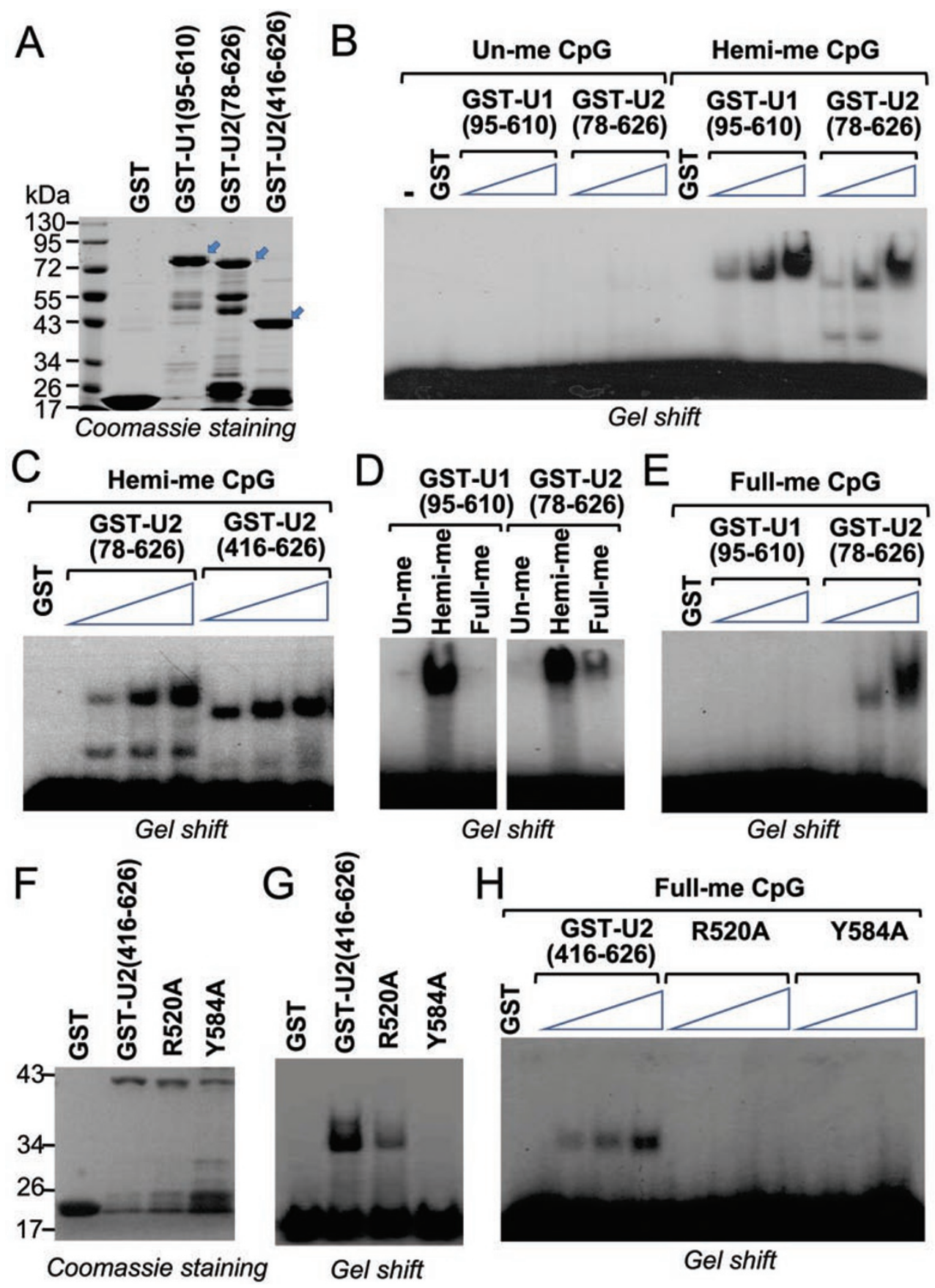

Figure 2 UHRF2 binds hemi- and fully methylated DNA through its SRA domain. (A) Coomassie blue staining gel showing purified GST-fusions of UHRF1 and UHRF2. The regions of UHRF1 or UHRF2 in number of amino acids fused to GST were as indicated. Arrows indicate the positions of GST-fusion proteins with the expected sizes. Also indicated are protein size markers. (B) Gel mobility shift assay showing binding of GST-UHRF1 (95-610) and GST-UHRF2 (78-626) to hemi-methylated but not un-methylated DNA probes. An increasing amount of recombinant proteins $(0.25 \mu \mathrm{g}, 0.5 \mu \mathrm{g}$ and $1 \mu \mathrm{g})$ were used for gel shift. (C) The recombinant UHRF2 containing SRA domain alone, GST-UHRF2 (416-626), is capable of binding hemi-methylated DNA probe. $0.25,0.5$ and $1 \mu \mathrm{g}$ of proteins were used for gel shift. (D) Comparison of GST-UHRF1 (95-610) and GST-UHRF2 (78-626) in binding of unmethylated, hemi-methylated (one strand methylated) and fully methylated (both strands methylated) DNA probe. (E) Increasing amount of GST-UHRF1 (95-610) and GST-UHRF2 (78-626) were compared for binding of fully methylated DNA probe. Three concentrations used were $0.25,0.5$ and $1 \mu \mathrm{g}$, respectively. (F) Coomassie blue staining gel showing purified GST, GST-UHRF2 (416626), and GST-UHRF2 (416-626) with R520A or Y584A mutation. (G) Gel mobility shift assay showing impaired hemi-methylated DNA binding activity for R520A and Y584A mutants. (H) R520A and Y584A mutants also showed impaired binding activity for fully methylated DNA probe. Three concentrations of proteins used were $0.25,0.5$ and $1 \mu \mathrm{g}$, respectively. 


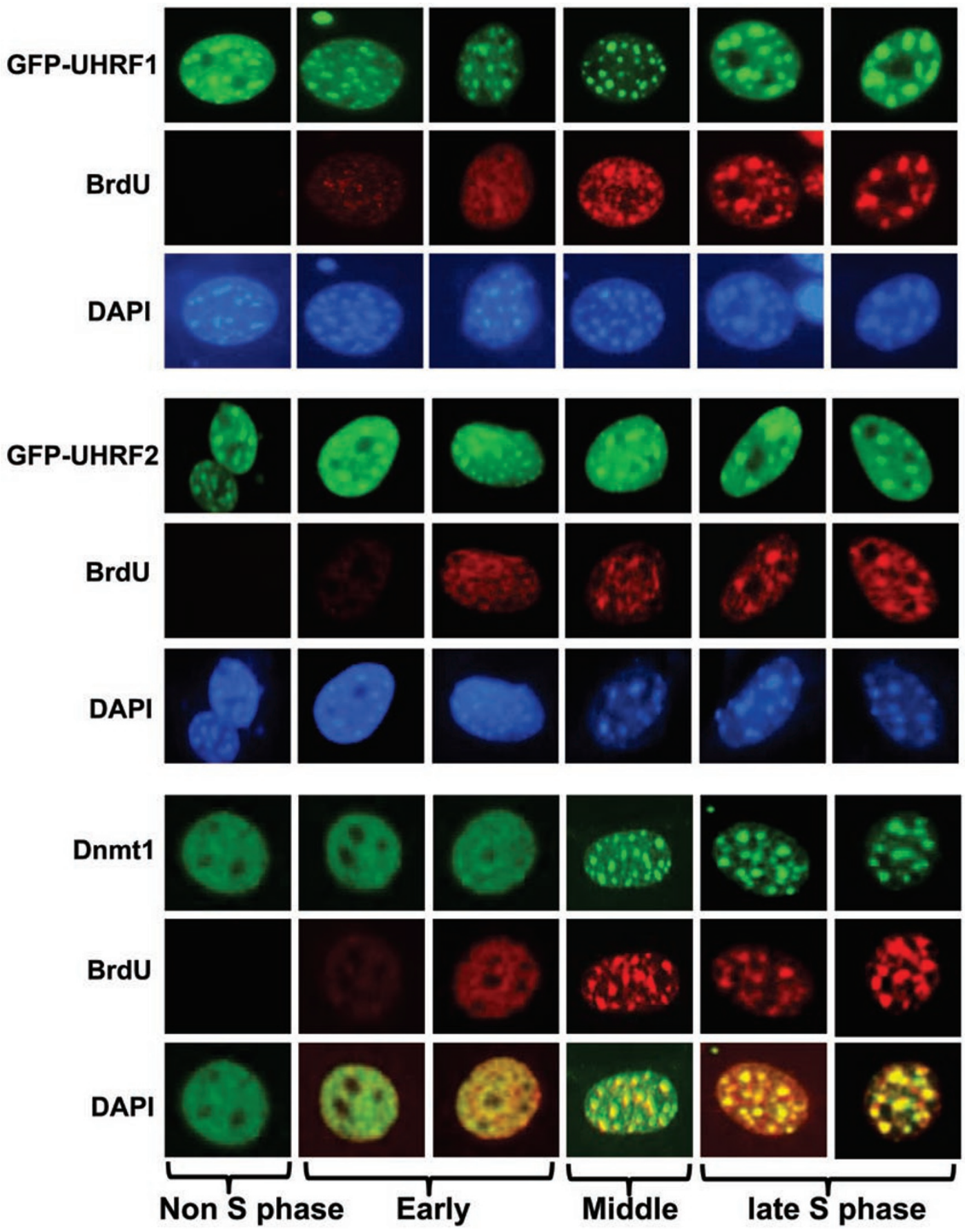

Figure 3 UHRF1 and UHRF2 are enriched at pericentric heterochromatins independent of cell cycle, whereas DNMT1 is enriched at pericentric heterochromatins only in middle and late S phase. NIH3T3 cells were transfected with GFP-UHRF1 or GFP-UHRF2 and blocked at S phase by aphidicolin treatment. The cells were then released from $S$ phase block and labeled with BrdU (red) and also stained with 4',6-diamidino-2-phenylindole (DAPI). DNMT1 was revealed by immunostaining using a DNMT1 specific antibody. The DAPI staining foci represent pericentric heterochromatins.

erochromatin association, we analyzed the subcellular localization of wild-type and UHRF2 mutants deficient in methylated $\mathrm{H} 3 \mathrm{~K} 9$ and/or methylated DNA binding in
NIH3T3 cells. For wild-type UHRF2, we observed colocalization with bright DAPI foci in approximately $75 \%$ transfected cells and diffuse nuclear staining in $\sim 25 \%$ of 
cells in more than 300 transfected cells (Figure 4B). For WF143/144AA and FW262/263AA mutants defective in binding $\mathrm{H} 3 \mathrm{~K} 9 \mathrm{me} 2 / 3$, the colocalization with DAPI foci was observed in only $\sim 4.7 \%$ and $3.6 \%$ cells, respectively, suggesting that the methylated H3K9-binding activity is crucial for UHRF2 pericentric heterochromatin localization (Figure 4B). For R520A and Y584A mutants in the full-length UHRF2 context, the colocalization with DAPI foci is also reduced (dropping to $\sim 18 \%$ and $\sim 23 \%$, respectively), but the reduction is less severe compared to the H3K9me2/3-binding deficient mutants (Figure 4B). These results suggest that the H3K9me2/3-binding activity is critically important for UHRF2's heterochromatin association, whereas the methylated DNA-binding activity also contributes. Similar results were obtained in multiple independent experiments. Taken together, we conclude that the binding activities for both methylatedH3K9 and methylated DNA function cooperatively to target UHRF2 to pericentric heterochromatin.
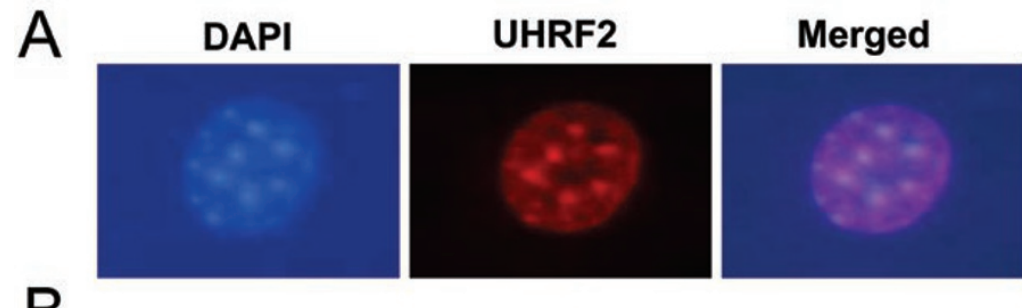

\section{Endogenous \\ UHRF2 \\ $70 \% \mathrm{HC}$}
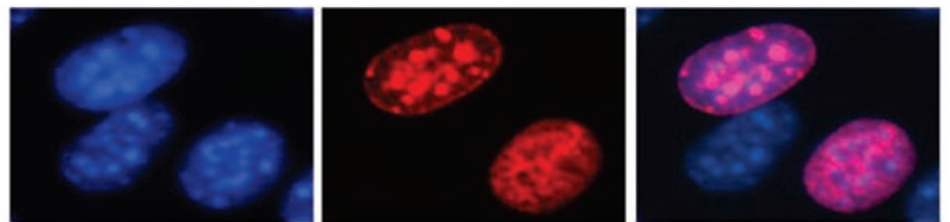

Flag-UHRF2

$74.9 \% \mathrm{HC}$

H3mK9(+)

Hemi-mCpG (+)
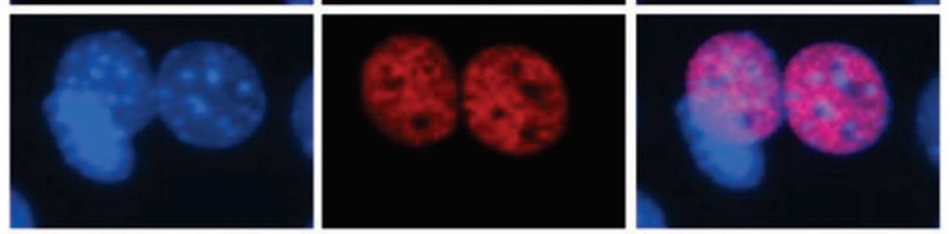

WF143/144AA

$4.7 \% \mathrm{HC}$

H3mK9(-)

Hemi-mCpG (+)
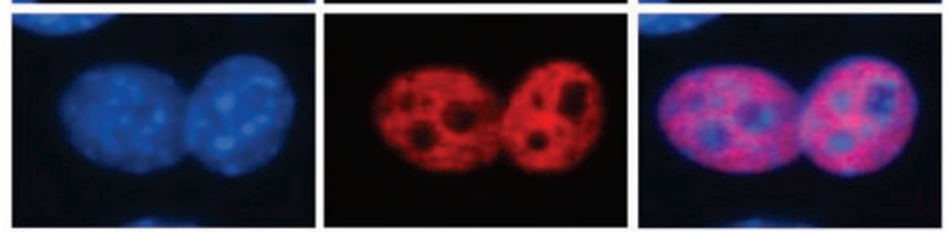

FW262/263AA

$3.6 \% \mathrm{HC}$

H3mK9(-)

Hemi-mCpG (+)
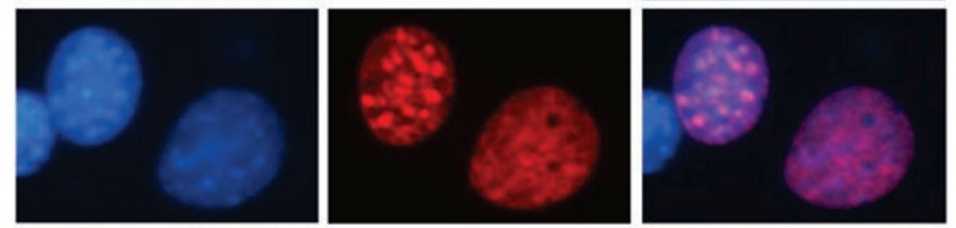

\section{A}

$17.8 \% \mathrm{HC}$

H3mK9(+)

Hemi-mCpG (-)
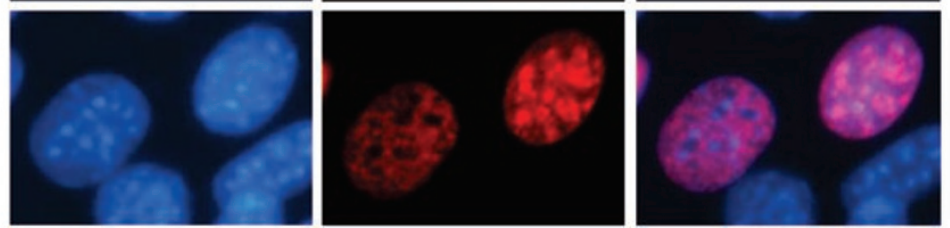

Y584A

$22.6 \%$ HC

H3mK9(+)

Hemi-mCpg (-)

Figure 4 The $\mathrm{H} 3 \mathrm{~K} 9 \mathrm{me} 2 / 3$ binding activity is the primary determinant for pericentric heterochromatin association of UHRF2. (A) The pericentric heterochromatin (HC) association for endogenous UHRF2 in NIH3T3 cells. (B) The pericentric heterochromatin localization for wild-type, methylated H3K9-binding deficient (WF143/144AA and FW262/263AA) and hemi-methylated DNA binding deficient (R520A and Y584A) mutants was analyzed in NIH3T3 using anti-Flag antibody cells. After counting large quantity of cells, the percentage of cells with UHRF2 and DAPI co-localization with DAPI foci representing pericentric heterochromatin (HC) was calculated for wild-type UHRF2 and each mutant. 
No protein-protein interaction between UHRF1 and UHRF2

Given that members of the same protein family are often found to interact with each other to form heterodimers and that UHRF1 and UHRF2 share the same cellular localization, we next tested whether UHRF1 and UHRF2 interact with each other. We first cotransfected 293 T cells with a Flag-tagged UHRF1 and a GFP-tagged UHRF2 and carried out coimmunoprecipitation analysis. A representative result in Figure 5A shows that there is no coimmunoprecipitation between Flag-tagged UHRF1 and GFP-tagged UHRF2. To test the interaction in cells, we made use of a $\mathrm{CHO}$ cell line, which contains large numbers of Lac operator sequences stably integrated in a chromosomal site [34]. Expression of CFP-tagged Lac-
UHRF1 fusion proteins in these cells generated bright foci due to the binding of CFP-Lac-fusion proteins to the locus with large numbers of Lac sequences. UHRF2 was expressed as a Flag-tagged protein and its interaction with UHRF1 was examined by colocalization with CFPLac-UHRF1 through immunostaining using an anti-Flag antibody. The result in Figure 5C shows that there is no colocalization of CFP-Lac-UHRF1 and Flag-UHRF2. Similarly, the colocalization was not observed when we did the reverse experiment using GFP-Lac-UHRF2 and Flag-UHRF1. Based on these two experiments, we conclude that UHRF1 and UHRF2 do not appear to interact with each other. We also tested whether UHRF2 may form homodimers or oligomers by testing the coimmunoprecipitation between GFP-UHRF2 and Flag-

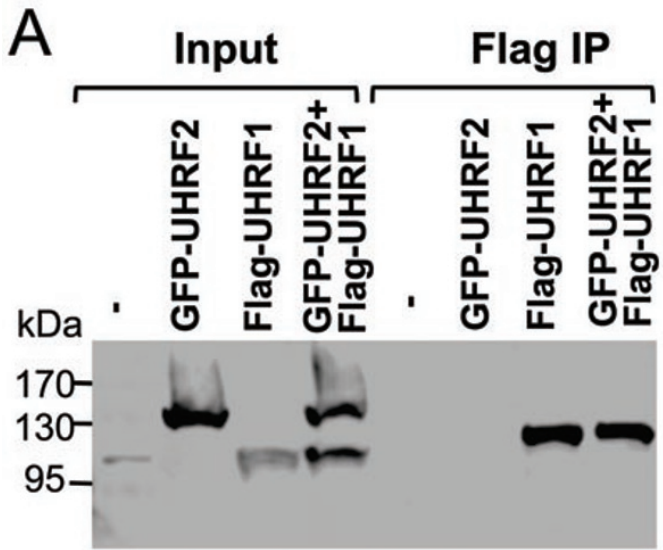

WB:UHRF1+UHRF2
C
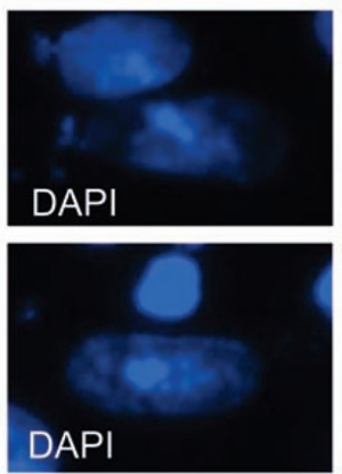
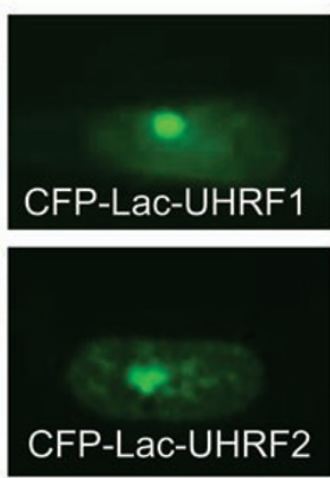

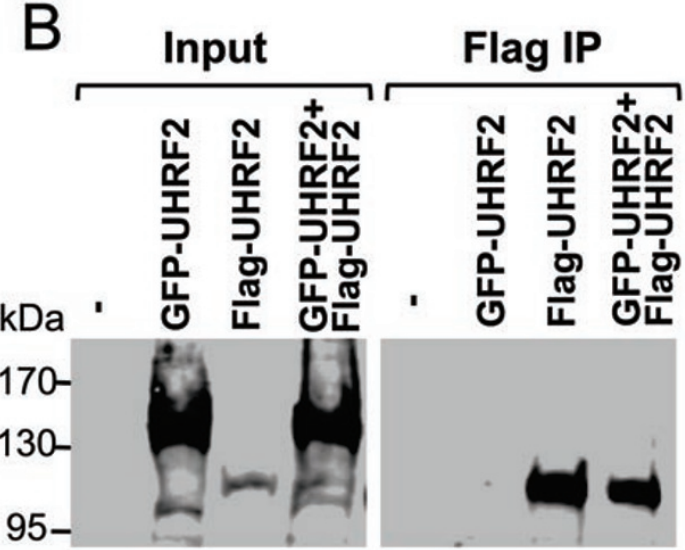

WB: UHRF2
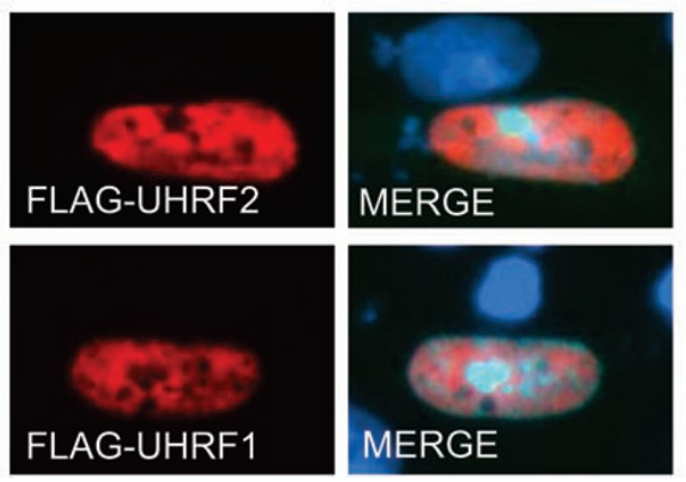

Figure 5 UHRF2 forms neither heterodimers with UHRF1 nor homodimers with itself. (A) UHRF2 did not coimmunoprecipitate with UHRF1. The interaction between UHRF1 and UHRF2 was tested via coimmunoprecipitation between GFP-UHRF2 and Flag-UHRF1 transiently expressed in 293T cells. (B) UHRF2 did not form homodimers or oligomers. The interaction between UHRF2 and UHRF2 was tested via coimmunoprecipitation between GFP-UHRF2 and Flag-UHRF2 transiently expressed in 293T cells. (C) No co-localization between UHRF1 and UHRF2 was observed in DG44-CHO cells. As DG44-CHO cells contain hundreds and thousands of Lac operons integrated in a single genomic site, expression of CFP-Lac-UHRF1 or CFP-Lac-UHRF2 all led to the observation of a bright CFP foci. However, Flag-UHRF2 was not recruited to the bright CFPLac-UHRF1 foci and Flag-UHRF1 was not recruited to the bright CFP-Lac-UHRF2 foci. 


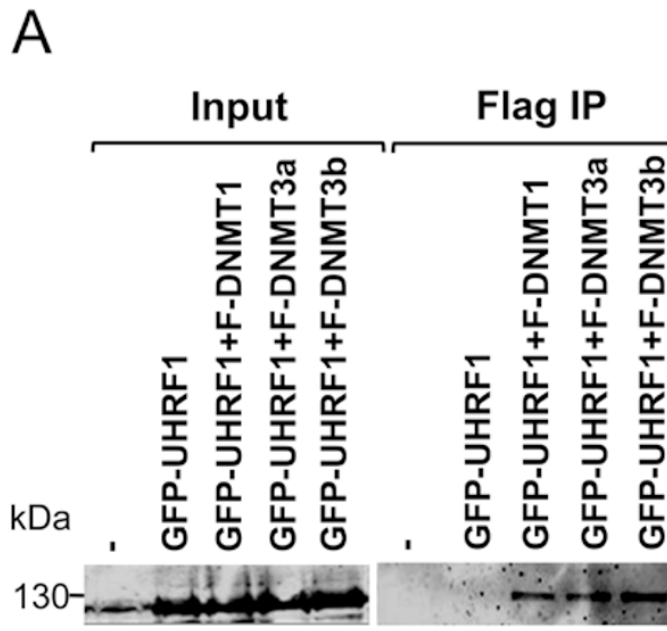

WB: $\alpha$ UHRF1

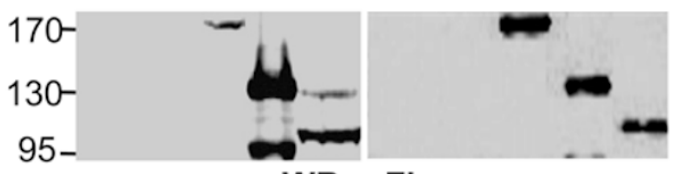

WB: $\alpha$ Flag

C

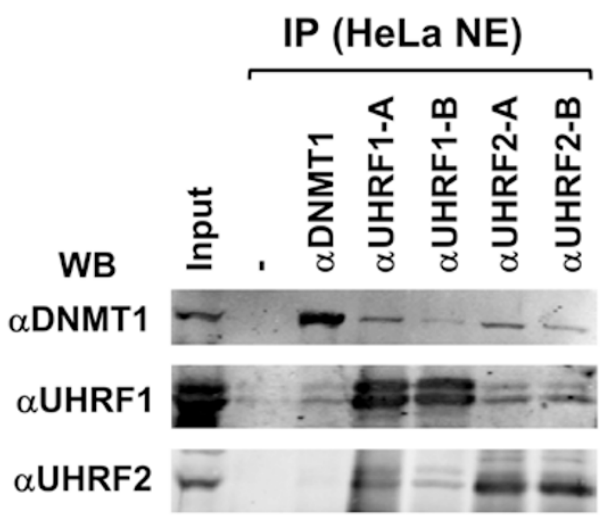

B

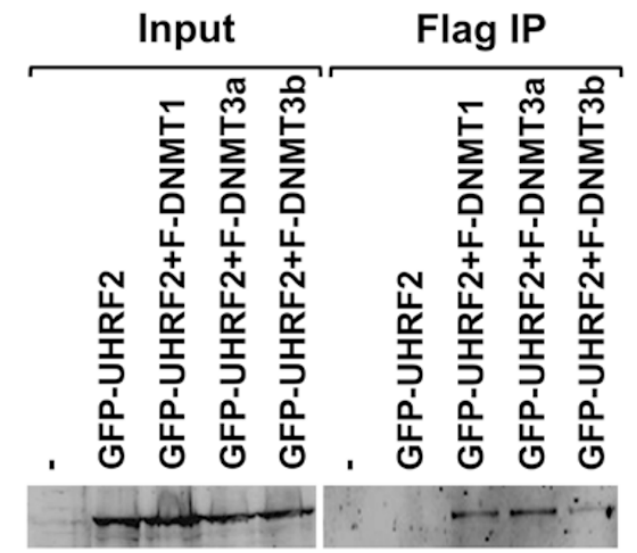

WB: $\alpha$ UHRF2

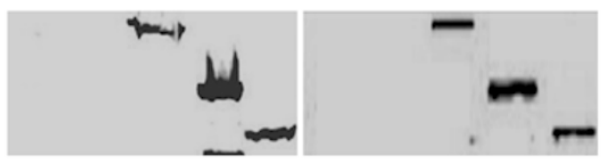

WB: $\alpha$ Flag

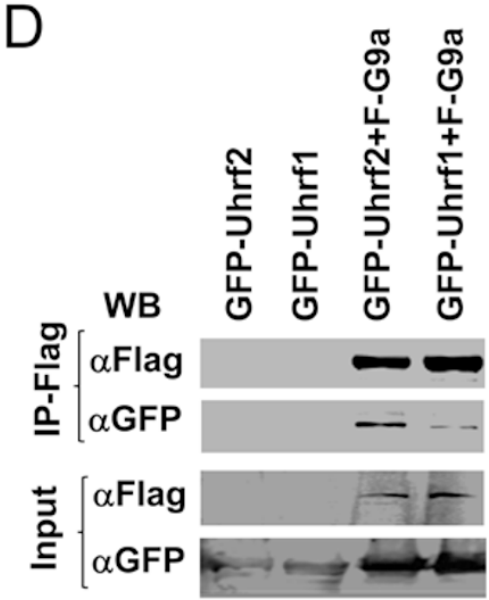

Figure 6 UHRF2 interacts with DNMT1, DNMT3a, DNMT3b and G9a. (A) UHRF1 co-immunoprecipitated with DNMT1, DNMT3a and DNMT3b. GFP-tagged UHRF1 was expressed alone or together with DNMT1, DNMT3a and DNMT3b, respectively in 293T cells and subjected to immunoprecipitation analysis using anti-Flag antibody and western blot analysis using UHRF1 and Flag antibodies. (B) UHRF2 co-immunoprecipitated with DNMT1, DNMT3a and DNMT3b. The co-immunoprecipitation experiments were performed as above except GFP-UHRF2 was used. (C) UHRF1 and UHRF2 in HeLa nuclear extracts coimmunoprecipitated with DNMT1. Note that low levels of UHRF1 were detected in immunoprecipitation of UHRF2 and vice versa, most likely due to slight cross-reaction in western blot between UHRF1 and UHRF2 antibodies. (D) Both UHRF1 and UHRF2 co-immunoprecipitated with G9a. GFP-tagged UHRF2 or UHRF1 were expressed alone or together with Flag-G9a in 293T cells and subjected to IP-western analysis using antibodies as indicated.

UHRF2. Again, we found no evidence for the formation of UHRF2 homodimers or oligomers (Figure 5B).

UHRF2 interacts with DNMT1, DNMT3a, DNMT3b and G9a

Previous studies have shown that UHRF1 interacts with DNMT1, DNMT3a, DNMT3b and G9a and through these interactions UHRF1 have been implicated in regulating DNA methylation maintenance, de novo DNA methylation and transcriptional repression $[8,35,36]$. We thus tested if UHRF2 also interacts with these proteins. To this end, we cotransfected 293T cells with GFP- 
tagged UHRF1 and Flag-tagged DNMT1, DNMT3a and DNMT3b, respectively and confirmed by co-immuno- precipitation the interaction of UHRF1 with DNMT1, DNMT3a and DNMT3b (Figure 6A). We then carried

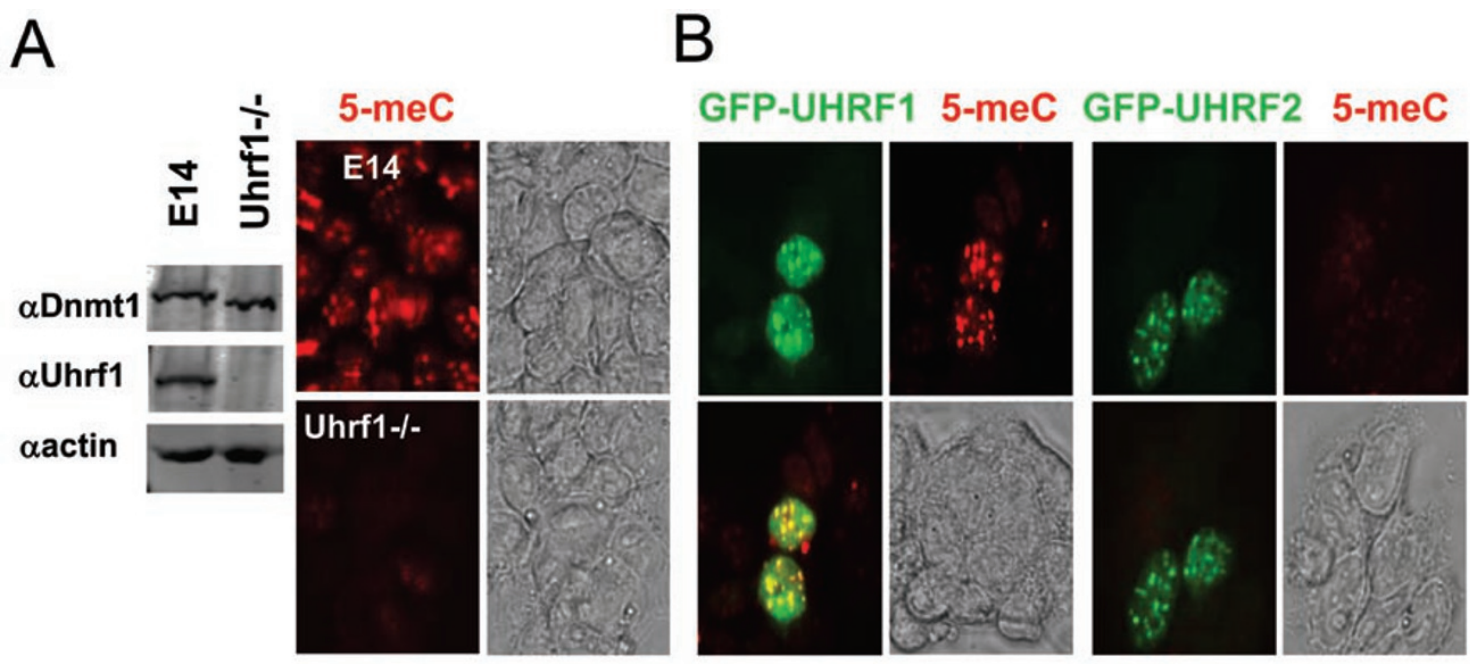

C

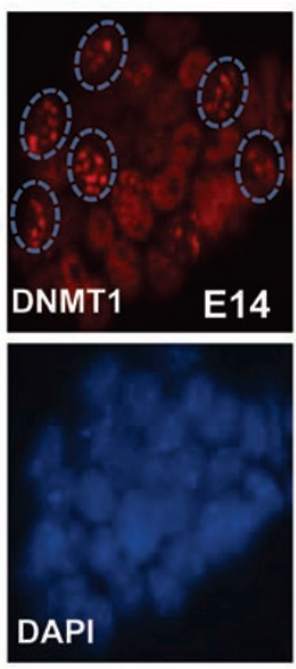

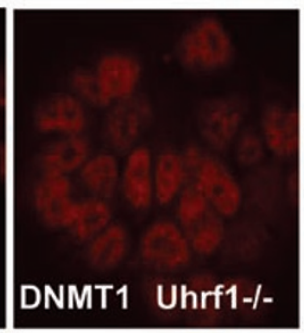

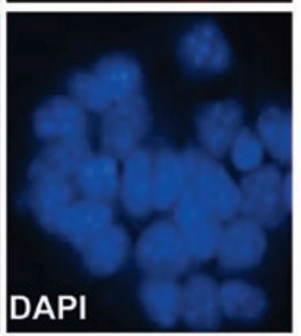

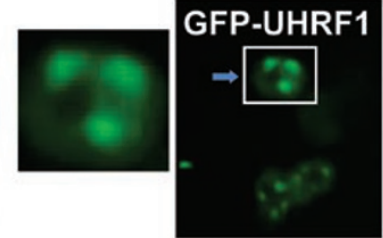
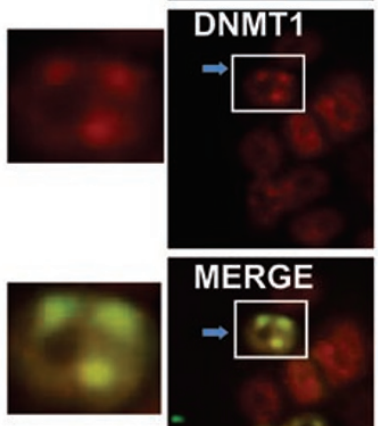

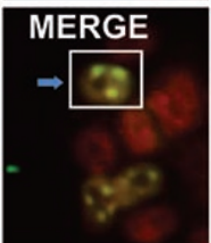

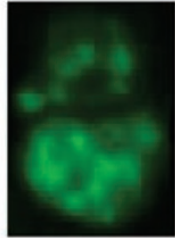
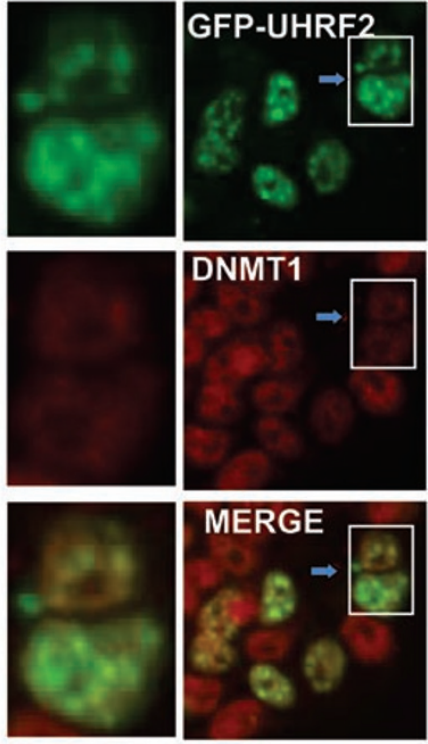

Figure 7 Overexpression of UHRF2 in mouse Uhrf1-/- ES cells rescues neither DNA methylation defect nor pericentric heterochromatin localization of DNMT1 in S phase. (A) Confirmation of DNA methylation defect in Uhrf1-/- ES cells by anti$\mathrm{mC}$ immunostaining. Left panel shows western blot analysis of whole cell extracts derived from wild-type E14 and Uhrf1-/ES cells. Right panel shows representative 5-meC immunostaining data for E14 and Uhrf1-/- ES cells. Also shown are the phase contrast images. (B) Ectopic expression of GFP-UHRF1 but not GFP-UHRF2 rescued DNA methylation defect in Uhrf1-/- ES cells. Note both GFP-UHRF1 and GFP-UHRF2 exhibited a focal staining pattern, in agreement with their expected pericentric heterochromatin localization. (C) Immunostaining for DNMT1 confirmed absence of focal staining pattern (pericentric heterochromatin targeting) for DNMT1 in Uhrf1-/- cells. The cells with DNMT1 focal staining in E14 were circled. Also shown are DAPI staining images. (D) Expression of GFP-UHRF1 but not GFP-UHRF2 restored correct pericentric heterochromatin targeting of DNMT1. The Uhrf1-l- ES cells were transfected with GFP-UHRF1 or GFP-UHRF2 and then subjected to immunostaining for DNMT1. The merged image revealed the same focal localization patterns for DNMT1 and GFPUHRF1. Left panel shows enlarged images of the cells marked by rectangle. Note that no focal staining pattern for DNMT1 was observed for GFP-UHRF2 expressing cells. 
out the same experiments with GFP-UHRF2 and demonstrated that GFP-UHRF2 also co-immunoprecipitated with Flag-DNMT1, DNMT3a and DNMT3b (Figure 6B). To test the interaction with DNMT1 further, IP-western analysis with HeLa nuclear extracts was performed. We used two anti-UHRF1 and two anti-UHRF2 antibodies. As shown in Supplementary information, Figure S2, these antibodies are specific for IP but showed certain levels of cross-reaction in western blot. The results in Figure $6 \mathrm{C}$ show that antibodies against both UHRF1 and UHRF2 co-precipitated DNMT1. Together these results demonstrate that, like UHRF1, UHRF2 also shows interaction with DNMT1 in co-immunoprecipitation assays. We also tested the potential interaction between UHRF2 and G9a, and IP-western results in Figure 6D show that both UHRF1 and UHRF2 co-immunoprecipitated with G9a.

\section{UHRF 2 cannot rescue DNA methylation defect in} Uhrf1-/-ES cells

So far our data show that, just like UHRF1, UHRF2 also recognizes specifically hemi-methylated DNA and interacts with DNMT1. Given that Uhrf1-/- mouse ES cells have severely reduced DNA methylation $[7,8]$, we tested whether ectopic overexpressing UHRF2 would rescue DNA methylation in Uhrf1-/- ES cells. We first confirmed by immunostaining using an anti-methyl $\mathrm{C}$ antibody that in comparison to the wild type E14 ES cells, the level of DNA methylation is substantially lower in the Uhrf1-/- ES cells (Figure 7A). We also confirmed by western blot the UHRF1 protein was absent in the Uhrf1-/- ES cells (Figure 7A). We then expressed GFPUHRF1 and GFP-UHRF2 in the Uhrf1-/- ES cells and examined the DNA methylation status by immunostaining using anti-methyl $\mathrm{C}$ antibody. As shown in Figure $7 \mathrm{~B}$, we found that while expression of GFP-UHRF1 rescued the DNA methylation defects in Uhrfl-/- ES cells, no rescue of DNA methylation was observed for cells expressing GFP-UHRF2. Robust rescue of DNA methylation by GFP-UHRF1 can be detected by immunostaining $48 \mathrm{~h}$ after transfection and further, the extended culture did not enhance significantly the levels of DNA methylation. No rescue of DNA methylation was observed even for prolonged culture after transcfection of GFP-UHRF2 (Figure 7B), Flag-UHRF2 or non-tagged UHRF2 (data not shown). These results demonstrate that UHRF2 is unable to substitute for UHRF1 in DNA methylation maintenance. Consistent with a non-essential role for UHRF2 in DNA methylation maintenance, we found that knockdown of UHRF2 in HCT116 cells by two different siRNAs did not lead to reduced levels of DNA methylation when examined by immunostaining using anti-methyl $\mathrm{C}$ antibody, whereas knockdown of UHRF1 led to reduced levels of DNA methylation (Supplementary information, Figure S3). These results were further supported by analyzing the levels of DNA methylation by more quantitative HPLC analysis (Li et al., unpublished data).

\section{UHRF2 could not target DNMT1 to replication foci in $S$ phase}

Previous studies indicate that DNMT1 could not be appropriately targeted to the DNA replication foci during $\mathrm{S}$ phase of the cell cycle in Uhrf1-/- ES cells [8]. We confirmed that in the wild-type E14 cells, DNMT1 could be observed in discrete foci in 10-15\% cells (Figure 7C). We confirmed by EdU labeling the cells with discrete DNMT1 foci were also positive for EdU labeling and belonged to cells in S phase (data not shown). In agreement with previous result [8], no discrete DNMT1 foci were observed in the Uhrf1-/- ES cells (Figure 7C), confirming a defect in targeting DNMT1 to replicating foci in these cells. We thus examined whether ectopic expression of UHRF1 and UHRF2 could restore the proper foci staining of DNMT1. As shown in Figure 7D, we found that expression of GFP-UHRF1 was able to restore DNMT1 foci staining patterns in approximately $10 \%$ of the cells (in S phase). However, no DNMT1 foci staining could be observed in the GFP-UHRF2expressing Uhrf1-/- ES cells. Note that both GFPUHRF1 and GFP-UHRF2 exhibit a discrete foci-staining pattern (Figure 7D), indicating both of them are properly localized to the pericentric heterochromatin foci. These results indicate that although both UHRF1 and UHRF2 localize to pericentric heterochromatin foci, only UHRF1 but not UHRF2 is able to recruit DNMT1 to these foci in $\mathrm{S}$ phase of the cell cycle, thus providing a mechanistic explanation for UHRF2's inability to rescue the DNA methylation defect in Uhrf1-/- ES cells.

\section{Difference in $S$ phase-dependent interaction with DNMT1 sets apart UHRF1 and UHRF2}

Given that both UHRF1 and UHRF2 interact with DNMT1 in coimmunoprecipitation experiments, we were surprised that only UHRF1, but not UHRF2, is able to recruit DNMT1 to DNA replication foci and thus plays a role in DNA methylation maintenance. As reported previously and shown in Figures 3 and 4, both UHRF1 and UHRF2 localize to pericentric heterchromatin foci in majority of NIH3T3 cells, yet DNMT1 is recruited and colocalizes with UHRF1 only in the S phase of the cell cycle, indicating an S phase-dependent interaction. For endogenous UHRF1 in mouse ES E14 cells, pericentric heterochromatin localization is clearly detected for the cells in $\mathrm{S}$ phase, but less obvious for 


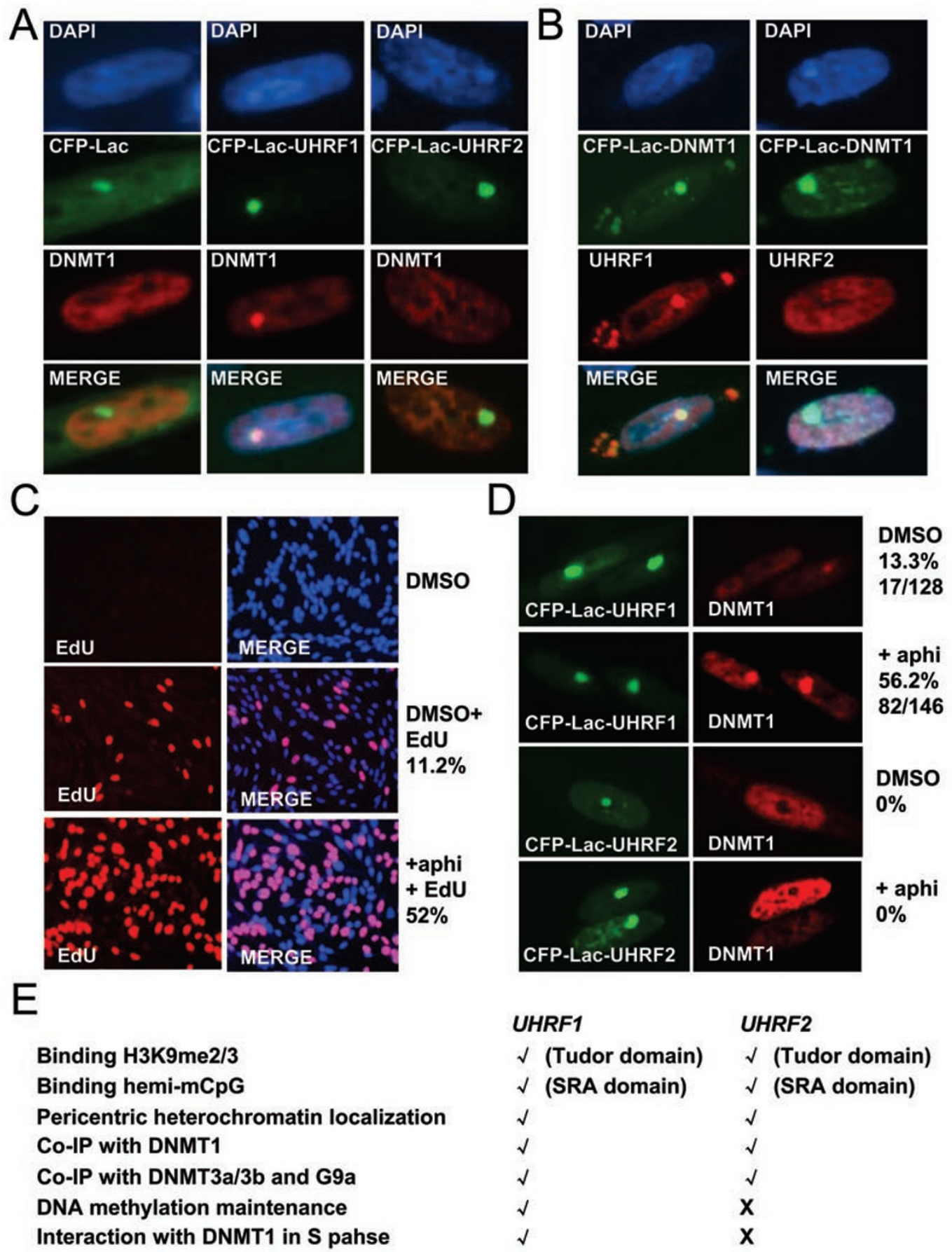

Figure 8 UHRF1 but not UHRF2 interacts with DNMT1 in DG44-CHO cells and this interaction is S phase dependent. (A) The interactions between DNMT1 and UHRF1, and DNMT1 and UHRF2 were analyzed in DG44-CHO cells using CFPLac-UHRF1, CFP-lac-UHRF2 and Flag-DNMT1. Expression of control CFP-Lac, CFP-Lac-UHRF1 and CFP-Lac-UHRF2 all resulted in a bright foci in cells. However, recruitment of Flag-DNMT1 was observed only for CFP-Lac-UHRF1 cells in about 10\% - 15\% cells expressing both CFP-Lac-UHRF1 and Flag-DNMT1. (B) The interaction with DNMT1 was reciprocally analyzed using CFP-Lac-DNMT1 and Flag-UHRF1 and Flag-UHRF2 in DG44-CHO cells. The colocalization with CFP-LacDNMT1 was observed only for Flag-UHRF1 but not Flag-UHRF2. Note the colocalization was also observed on other regions enriched with CFP-Lac-UHRF1. This interaction is again observed only in 10\% - 15\% cells coexpressing both CFP-Lac-DNMT1 and Flag-UHRF1. (C) Aphidicolin treatment enriched DG44-CHO cells in S phase. DG44-CHO cells were treated with or without $1 \mathrm{mg} / \mathrm{ml}$ aphidicolin for $20 \mathrm{~h}$. Aphidicolin was removed and cells were incubated in the presence of $10 \mu \mathrm{M}$ EdU for $2 \mathrm{~h}$ and processed for EdU immunostaining (left panel) and merged figures with DAPI staining (right panel). (D) Aphidicolin treatment similarly increased the DG44-CHO cells with UHRF1 and DNMT1 colocalization. 
the cells in other phases of the cell cycle, likely due to cell cycle-dependent expression and protein stability of endogenous UHRF1 [8]. To circumvent this problem, we analyzed whether the interaction of DNMT1 with UHRF1 and UHRF2 is also S phase-dependent in CHO cells. As shown in Figure 8A, the colocalization of CFPLac-UHRF1 and Flag-DNMT1 was detectable, but in only about $10 \%$ of the cells expressing both proteins. However, under the same condition, no colocalization of CFP-Lac-UHRF2 and Flag-DNMT1 was observed in large numbers of cells expressing both proteins. The same results were observed when the experiments were performed reciprocally using CFP-Lac-DNMT1 and Flag-UHRF1 and Flag-UHRF2 (Figure 8B).

The above results that UHRF1 and DNMT1 were colocalized in only $\sim 10 \% \mathrm{CHO}$ cells suggest that the interaction between these two proteins in $\mathrm{CHO}$ cells likely occurs only in the cells in S phase. To demonstrate this further, we treated the cells with aphidicolin to enrich the cells in S phase. EdU immunostaining in Figure 8C showed that aphidicolin treatment increased the cells in S phase from $11.2 \%$ to $52 \%$. Importantly, we found that aphidicolin treatment increased the cells with CFP-LacUHRF1 and Flag-DNMT1 colocalization from 13.3\% to $\sim 56.2 \%$ (Figure $8 \mathrm{D}$ ), which correlates very well with the percentage of the cells in S phase in the population. These results argue strongly that the interaction between UHRF1 and DNMT1 in CHO cells is cell cycle-dependent and occurs only during $\mathrm{S}$ phase of the cell cycle. However, under the same conditions we did not observe any colocalization between DNMT1 and UHRF2 (Figure 8D). Thus, both in ES cells and CHO cells, the interaction between DNMT1 and UHRF1 is S phase-dependent. Although both UHRF1 and UHRF2 interact with DNMT1 in coimmunoprecipitation assays, it is the lack of S phase-dependent interaction with DNMT1 that sets apart UHRF2 from UHRF1.

\section{Discussion}

Both UHRF1 and UHRF 2 possess $H 3 \mathrm{~K} 9 \mathrm{me} 2 / 3$ and hemi-methylated DNA binding activities

In this study we show that, much like UHRF1, UHRF2 also has both $\mathrm{H} 3 \mathrm{~K} 9 \mathrm{me} 2 / 3$ and hemi-methylated DNA binding activities. Also like UHRF1, UHRF2 exhibits predominantly a constitutive pericentric heterochromatin localization on top of a diffuse nuclear distribution. We mapped the H3K9me2/3-binding activity to its tudor domain, as mutations of key amino-acid residues in the tudor domain abolish the methylated H3K9 binding activity (Figure 1). Although it is less obvious for UHRF1 and UHRF2 in HeLa nuclear extracts, in vitro synthesized
UHRF2 and recombinant UHRF2 containing both tudor and PHD domains also exhibited a weak binding activity for unmodified $\mathrm{H} 3$ peptide (Figure 1C and 1D). This observation is consistent with recent reports that the PHD domain of UHRF1 can independently recognize unmodified $\mathrm{H} 3$ peptide. However, it remains to be determined whether the PHD domain and tudor domain of UHRF2 function as independent histone $\mathrm{H} 3$ binding modules and/or as a reader for combinatorial code of unmodified (H3R2) and H3K9me2/3 in cells.

Much like UHRF1, UHRF2 also binds hemi-methylated DNA (Figure 2). By using the same DNA probe that was unmethylated, hemi-methylated or fully-methylated we demonstrated that both UHRF1 and UHRF2 have a strong preference for hemi-methylated DNA and exhibit virtually no binding for un-methylated DNA (Figure 2B). Interestingly, we observed that UHRF2 also bound moderately to fully methylated probe, whereas UHRF1 did not (Figure 2D and 2E). The binding activity for both hemi- and fully methylated DNA requires its conserved SRA domain, as mutations of key residues in the SRA domain abolish both binding activities (Figure $2 \mathrm{G}$ and $2 \mathrm{H})$. The finding that UHRF2 binds fully methylated DNA better than UHRF1 suggests that UHRF2 may bind fully methylated $\mathrm{CpG}$ in cells and play a role in transcriptional regulation involving methylated DNA. Future work is required to assess the relative methyl-CpG binding activity of UHRF2 by comparing it with other known methyl-CpG binding proteins such as MeCP2.

During the review of our manuscript, Pichler et al. reported coorperative binding of methylated $\mathrm{H} 3 \mathrm{~K} 9 \mathrm{me} 3$ and hemi-methylated DNA by UHRF2 [37]. They used a GFP fusion protein-based in vitro binding assay to characterize the binding of histone tail peptides and methylated DNA. In their study, the relative binding affinity for $\mathrm{H} 3 \mathrm{~K} 9 \mathrm{me} 3$ by UHRF2 is not $50 \%$ more than that for unmodified $\mathrm{H} 3$ peptide. Similarly, the binding affinity for hemi-methylated DNA by UHRF2 is not $50 \%$ more than that of unmethylated DNA. In contrast, in our pulldown assays using either HeLa nuclear extracts, in vitro translated proteins or recombinant GST-fusion proteins purified from bacteria, we consistently observed a robust preference for binding of H3K 9 me $2 / 3$ over H3. Similarly, in our gel mobility shift-based assays, recombinant UHRF2 exhibited a strong preference for binding of hemi- $>$ fully- $>$ unmethylated DNA probe. Such discrepancy may result from the difference in assay systems, fusion proteins used and DNA probe sequences.

Currently, UHRF1 and UHRF2 are the only proteins that are able to bind both methylated $\mathrm{H} 3 \mathrm{~K} 9$ and DNA. Both UHRF1 and UHRF2 are enriched in the heavy DAPI stained pericentric heterochromatin foci, 
which contain high levels of both methylated $\mathrm{H} 3 \mathrm{~K} 9$ and DNA. In this regard, Pichler et al. provided evidence that UHRF 2 binds cooperatively $\mathrm{H} 3 \mathrm{~K} 9 \mathrm{me} 3$ and hemimethylated DNA [37]. We find that the methylated H3K9-binding deficient UHRF2 mutants are severely impaired in heterochromatin localization, whereas mutations that impair its methylated DNA binding activity also affect its heterochromatin localization, although to a less extent (Figure 4). This observation is in agreement with the study by Pilchler et al. [37] showing that loss of H3K9me3 methylation has a more severe effect on UHRF2 pericentric heterochromatin localization than loss of DNA methylation. As methylated H3K9 and methylated DNA are often colocalized in the genome, UHRF1 and UHRF2 are likely to play a role in mediating the cross-talk between $\mathrm{H} 3 \mathrm{~K} 9$ methylation and DNA methylation.

UHRF2 cannot replace UHRF1 in DNA methylation maintenance and does not interact with DNMT1 in $S$ phase of the cell cycle

The ability for both UHRF1 and UHRF2 to bind methylated H3K9 and hemi-methylated DNA and the interaction with DNMT1 in co-IP raise an intriguing question as to whether UHRF2 also plays a role in DNA methylation maintenance. However, overexpression of UHRF2 could not rescue the DNA methylation defect in Uhrf1-/- ES cells. Similarly, Pichler et al. [37] reported that expression of mouse Uhrf2 in Uhrfl-/- ES cells did not restore DNA methylation at major satellite repeats. Furthermore, we showed that while knockdown of UHRF1 resulted in reduced DNA methylation in HCT116 cells, knockdown of UHRF2 in HCT116 cells did not lead to reduced levels of DNA methylation (Supplementary information, Figure S3). Similar results were obtained with knockdown of UHRF1 and UHRF2 in HeLa and NIH3T3 cells (data not shown). Thus, UHRF2 cannot substitute for UHRF1 in DNA methylation maintenance and does not appear to play a role in DNA methylation maintenance. Mechanistically, we found that while expression of UHRF1 rescued pericentric heterochromatin localization of DNMT1, expression of UHRF2 did not (Figure 7D). As shown in Figure 3, both exogenously expressed UHRF1 and UHRF2 localize to pericentric heterochromatin regions throughout the cell cycle, whereas DNMT1 shows a diffuse nuclear staining in early $\mathrm{S}$ phase and only colocalizes with UHRF1 in middle and late $\mathrm{S}$ phase. These results, together with the previous report [8], reveal that UHRF1 interacts with DNMT1 only during $\mathrm{S}$ phase of the cell cycle. In support of this, we demonstrate clearly that colocalization and thus the interaction between DNMT1 and UHRF1 in CHO cells is also $\mathrm{S}$ phase-dependent (Figure 8), indicating that the interaction between DNMT1 and UHRF1 is a tightly regulated event. Significantly, no S phase-dependent colocalization between DNMT1 and UHRF2 was observed under the same conditions. As the interaction between DNMT1 and UHRF1 is restricted to the S phase in cells, our data imply that DNA methylation maintenance exerted by DNMT1 is also restricted to the S phase of the cell cycle. This may have advantage to allow cells to couple the maintenance of DNA methylation with DNA replication and thus ensure the fidelity of epigenetic inheritance.

Currently it is not known why UHRF2 interacts with DNMT1 in coimmunoprecitation but not in S phase of the cell cycle. In addition, the mechanism(s) underlying the $\mathrm{S}$ phase-dependent interaction between UHRF1 and DNMT1 is unknown. One possibility is that the interaction between UHRF1 and DNMT1 is inhibited or masked by an unknown protein(s) that interacts with UHRF1 or DNMT1. During the S phase of the cell cycle, this inhibitory mechanism is relieved and thus the interaction between UHRF1 and DNMT1 is permitted. Alternatively, the interaction between UHRF1 and DNMT1 may require an $\mathrm{S}$ phase-dependent posttranslational modification, perhaps CDK2-dependent phosphorylation, of UHRF1 and/or DNMT1. For UHRF2, it does not interact with DNMT1 in S phase of the cell cycle either due to the presence of an inhibitory mechanism or due to the lack of an S phase-dependent modification required for DNMT1 interaction. In coimmunoprecitation assays preparation of cellular lysates may dislodge the inhibitory protein(s) or alter protein modification and thus result in interaction of DNMT1 with both UHRF1 and UHRF2.

Taken together, as summarized in Figure $8 \mathrm{E}$, our study demonstrates that biochemically UHRF2 behaves much like its paralogue UHRF1. Both proteins recognize methylated H3K9 and hemi-methylated DNA, and interact with DNMT1, DNMT3a, DNMT3b and G9a in vitro. Given that UHRF2 interacts with DNMT3a, DNMT3b and G9a, we speculate that UHRF2 may play a role in transcriptional regulation by targeting these proteins to chromatin regions containing methylated $\mathrm{H} 3 \mathrm{~K} 9$ and/ or methylated DNA. Future work will test whether this is the case. However, UHRF2 cannot target DNMT1 to replication foci in $\mathrm{S}$ phase and cannot substitute the role of UHRF1 in DNA methylation maintenance. Our study points to the cell-cycle-dependent interaction between UHRF1 and DNMT1 as a key regulatory mechanism targeting DNMT1 for DNA methylation. Future study is necessary to elucidate the molecular mechanism underlying the S phase-dependent interaction between UHRF1 and DNMT1. 


\section{Materials and Methods}

\section{Plasmids and antibodies}

The pSG5-Flag-UHRF1 was previously described [17]. The pSG5-Flag-UHRF2, pEGFP-C1-UHRF2, CFP-LacR-UHRF2 and pPYCAGIP-UHRF2 and pPYCAGIP-GFP-UHRF2 were generated by cloning the full-length human UHRF2 into each of the vectors. Similarly, pEGFP-C1-UHRF1, CFP-LacR-UHRF1 and pPYCAGIP-GFP-UHRF1 were generated by cloning full-length UHRF1 into these vectors. Deletion mutants and various GSTUHRF1 and GST-UHRF2 fusion constructs were generated by cloning the corresponding PCR products into pSG5-Flag vector and pGEX4T-1 vector, respectively. Point mutation mutants were generated by site-directed mutagenic PCR, according to the manufacturer's instruction.

The polyclonal UHRF1-A, UHRF1-B, UHRF2-A and UHRF2$B$ antibodies were raised against GST-UHRF1 (aa 386-650), GSTUHRF1 (aa 95-610), GST-UHRF2 (aa 1-416) and GST-UHRF2 (aa 416-691), respectively. The commercial antibodies used are PHF8 (AbMART), DNMT1 (AbMART), Flag (Sigma-Aldrich), HP1 $\alpha$ (Abcam), G9a (Abcam), GFP (Abcam) and methyl-C (Eurogentec).

In vitro binding of histone peptides with HeLa nuclear extracts, 35S-methionine-labeled proteins and recombinant proteins

Nuclear extracts were prepared from HeLa cells by the protocol of Dignam [38] and precleared with streptavidin-coated agarose beads. The various methylated histone $\mathrm{H} 3$ tail peptides contain the $\mathrm{H} 3$ amino acids 1-21 followed by a GGK linker sequence and a C-terminal biotin. The H4K20me2 peptide contains the $\mathrm{H} 4$ amino acids 11-30 followed by a GGK linker sequence and a C-terminal biotin. All peptides were synthesized and purified by Scilight Biotechnology LLC. For pull-down assays, approximately $1 \mu \mathrm{g}$ of histone tail peptides were first immobilized on streptavidin-coated beads, and after washing to remove the unbound peptide, they were incubated with HeLa cell nuclear extracts diluted once with binding buffer (20 mM HEPES ( $\mathrm{pH} 7.9$ ), $150 \mathrm{mM} \mathrm{KCl}, 1 \mathrm{mM}$ dithiothreitol (DTT), $1 \mathrm{mM}$ phenylmethylsulfonyl fluoride (PMSF), $10 \%$ glycerol, $0.1 \% \mathrm{NP}-40$, proteinase inhibitors) for $3 \mathrm{~h}$ at $4{ }^{\circ} \mathrm{C}$. Unbound proteins were removed by washing the beads with washing buffer (20 mM HEPES (pH 7.9), $150 \mathrm{mM} \mathrm{KCl}, 1 \mathrm{mM}$ DTT, $1 \mathrm{mM}$ PMSF, $0.1 \%$ NP-40, proteinase inhibitors) four times for 5 min each. The proteins that remained bound to the peptides were separated by SDS-PAGE followed by western blotting analysis.

For defining H3K9me2/3-binding domain of UHRF2, various 35S-methionine-labeled UHRF2 mutants were generated with the TNT-coupled reticulocyte lysate system (Promega). The reactions were diluted to $1: 10$ with the binding buffer and subjected to binding assays as above, except that the binding of UHRF2 proteins was revealed by autoradiography.

For determining direct binding of UHRF 2 to $\mathrm{H} 3 \mathrm{~K} 9 \mathrm{me} 2 / 3$, GST-UHRF1 and GST-UHRF2 were expressed and purified from E. coli. The binding of these purified proteins to control $\mathrm{H} 3$ and H3K 9 me $2 / 3$ peptides were essentially as described above, except for the presence of $0.2 \mathrm{mg} / \mathrm{ml} \mathrm{BSA}$ in the binding buffer. The binding of recombinant proteins was revealed by Coomassie blue staining.

\section{Gel mobility shift assay}

To prepare ${ }^{32} \mathrm{P}$-labeled unmethylated, hemi-methylated and full methylated DNA probes, $1 \mu \mathrm{g}$ of the forward and reverse strand of oligo either both unmethylated, only forward strand methylated or both strand methylated were mixed in $20 \mu 1$ annealing buffer, incubated at $68{ }^{\circ} \mathrm{C}$ for $15 \mathrm{~min}$ and then cooled gradually to room temperature. Approximately $50 \mathrm{ng}$ of annealed DNA was labeled with ${ }^{32} \mathrm{P}-\mathrm{dCTP}$ by fill-in reaction with klenow fragment in the presence of cold dATP, dTTP and dGTP. The free ${ }^{32} \mathrm{P}-\mathrm{dCTP}$ was removed by spin column and approximately 0.5 ng-labeled probe was used for each gel shift experiment. The sequences of forward and reverse strands of probe are 5'-AGCTGCG GGCAATCGAT

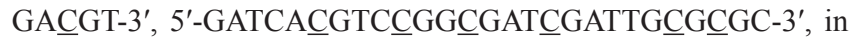
which the position of methyl-C was underlined.

For binding of recombinant GST fusion proteins, proteins ranging from 50 to $500 \mathrm{ng}$ were incubated with ${ }^{32} \mathrm{P}$-labeled probes in the binding buffer $(10 \mathrm{mM}$ Tris-Cl, $\mathrm{pH} 7.5,50 \mathrm{mM} \mathrm{NaCl}, 5 \mathrm{mM}$ $\mathrm{MgCl}_{2}, 1 \mathrm{mM}$ DTT, $0.05 \% \mathrm{NP}-40,5 \%$ glycerol, $50 \mathrm{ng} / \mu \mathrm{l}$ poly(dI$\mathrm{dC})$ ) at room temperature for $40 \mathrm{~min}$. For binding of full-length UHRF1 and UHRF2 purified from 293T cells, approximately 20 ng proteins were mixed with ${ }^{32} \mathrm{P}$-labeled probes essentially as above. The reactions were then electrophoresed on a $5 \%$ polyacrilamide gel in $0.5 \times \mathrm{TBE}$ buffer at $100 \mathrm{~V}$ for $0.5 \mathrm{~h}$. The results were visualized through autoradiography.

\section{Transient transfection, immunoprecipitation and western blot analysis}

Transient transfections of HEK-293, DG44-CHO and UHRF1-/- ES cells were carried out using Lipofectamine ${ }^{\mathrm{TM}} 2000$ (Invitrogen) essentially according to manufacturer's instruction. For testing protein-protein interaction by co-immunoprecipitation, HEK-293 cells were transfected with various plasmid combinations as indicated. $48 \mathrm{~h}$ after transfection, cells were collected, washed with PBS once and lysed with ice-cold lysis buffer (50 $\mathrm{mM}$ Tris- $\mathrm{HCl} \mathrm{pH}$ 7.4, $150 \mathrm{mM} \mathrm{NaCl}, 1 \%$ NP-40, 1 mM EDTA, $2 \mathrm{mM}$ PMSF, protease inhibitor cocktail from Sigma-Aldrich) for $30 \mathrm{~min}$ at $4{ }^{\circ} \mathrm{C}$ with occasional vortexing. The lysates were centrifugated for $10 \mathrm{~min}$ at $14000 \mathrm{rpm}$. The supernatants (whole cell extracts) were incubated with different antibodies and protein A-agarose beads or M2 anti-Flag beads (Sigma-Aldrich) for $5 \mathrm{~h}$ at $4{ }^{\circ} \mathrm{C}$. The beads were washed five times in TNEN buffer $(20$ $\mathrm{mM}$ Tris- $\mathrm{HCl} \mathrm{pH}$ 8.0, $100 \mathrm{mM} \mathrm{NaCl}, 1 \mathrm{mM}$ EDTA, 0.5\% NP-40, $1 \mathrm{mM}$ PMSF). Bound proteins were extracted with SDS-PAGE sample buffer, and analyzed on SDS-PAGE followed by western blot analysis.

Immunofluorescence staining, cell synchronization with aphidicolin and EdU labeling

NIH3T3 and Uhrf1-/- mouse ES cells were maintained with regular DMEM plus 10\% FBS and ES cell media routinely. For immunofluorescence staining of 5-meC and DNMT1, NIH3T3 or Uhrf1-/- ES cells were washed with $1 \times$ PBS prior to fixation in $4 \%$ paraformaldehyde. Cells for $5-\mathrm{meC}$ immunostaining were permeabilized with $4 \mathrm{M} \mathrm{HCl}$ at room temperature for $30 \mathrm{~min}$, whereas the cells for DNMT1 immunostaining were permeabilized with $1 \mathrm{M} \mathrm{HCl}$ on ice for $10 \mathrm{~min}$ and $2 \mathrm{M} \mathrm{HCl}$ at $37^{\circ} \mathrm{C}$ for $10 \mathrm{~min}$ once again. The cells were then neutralized with sodium borate $(\mathrm{pH} 9.0)$ at room temperature for $20 \mathrm{~min}$, blocked with $5 \% \mathrm{BSA}$ in a $37^{\circ} \mathrm{C}$ incubator for $15 \mathrm{~min}$ and incubated with 5-meC or DNMT1 antibody for $2 \mathrm{~h}$. For double immunostaining, the cells were incubated 
with the other one primary antibody, followed by incubation with Alexa Fluor 488 or Texas-Red-conjugated secondary antibodies. Images were acquired with a microscope system.

For examining co-localization in DG44-CHO cells, combinations of plasmids as indicated were transfected into DG44-CHO cells. One day after transfection, cells were processed for immunostaining using anti-Flag antibody. The images were acquired and examined for co-localization of CFP-Lac fusion with the Flagtagged protein of interest.

For enriching DG44-CHO cells in S phase, the cells were cultured with GIBCO medium supplemented with $1 \mathrm{mg} / \mathrm{ml}$ aphidicolin for $20 \mathrm{~h}$. To label the S phase DG44-CHO cells with EdU, the cells were washed and cultured with $10 \mu \mathrm{M}$ EdU for $2 \mathrm{~h}$ prior to fixation in $4 \%$ paraformaldehyde. Cells were then incubated with $2 \mathrm{mg} / \mathrm{ml}$ glycine in $37{ }^{\circ} \mathrm{C}$ incubator for $15 \mathrm{~min}$, permeabilized with $0.5 \%$ TritonX-100 on ice for $10 \mathrm{~min}$ and incubated with $1 \times$ Apollo reagent in a $37{ }^{\circ} \mathrm{C}$ incubator for $30 \mathrm{~min}$. The cells were also incubated with $1 \times$ Hoechst at room temperature for $20 \mathrm{~min}$ to label the nuclei. Images were acquired with a microscope system.

\section{Transient transfection, immunoprecipitation and western blot analysis}

HEK-293 cells were transfected by Lipofectamine ${ }^{\mathrm{TM}} 2000$ (Invitrogen) with various plasmid combinations as indicated. $48 \mathrm{~h}$ later, cells were washed with PBS (137 mM NaCl, $2.7 \mathrm{mM} \mathrm{KCl}$, $10 \mathrm{mM} \mathrm{Na}_{2} \mathrm{HPO}_{4}, 2 \mathrm{mM} \mathrm{KH} \mathrm{PO}_{4}$ ) and ice-cold lysis buffer (RIPA) (50 mM Tris- $\mathrm{HCl} \mathrm{pH} 7.4,150 \mathrm{mM} \mathrm{NaCl}, 1 \% \mathrm{NP}-40,1 \mathrm{mM}$ EDTA, 2 mM PMSF, protease inhibitor) was added. Cells were lysed for $30 \mathrm{~min}$ at $4{ }^{\circ} \mathrm{C}$ with occasional vortexing. The lysates were centrifugated for $10 \mathrm{~min}$ at $14000 \mathrm{rpm}$. The supernatants (whole cell extracts) were incubated with different antibodies and protein A-agarose beads or M2 beads (Sigma-Aldrich) for $5 \mathrm{~h}$ at $4{ }^{\circ} \mathrm{C}$. The beads were washed five times in TNEN buffer $(20 \mathrm{mM}$ Tris- $\mathrm{HCl}$ pH 8.0, 100 mM NaCl, 1 mM EDTA, 0.5\% NP-40, 1 mM PMSF). Bound proteins were extracted with SDS-PAGE sample buffer, and analyzed on SDS-PAGE followed by western blot analysis.

\section{Acknowledgments}

We like to thank Drs Guoliang Xu (Shanghai Institute of Biochemistry and Cell Biology, CAS, China), Bing Zhu (National Institute of Biological Sciences, China) and Guohong Li (Institute of Biophysics, Chinese Academy of Sciences, China) for generous support in reagents and valuable discussion. This study was partially supported by grants from the National Natural Science Foundation of China (90919025 and 30871381), the Ministry of Science and Technology of China (2009CB918402, 2009CB825601, 2010CB944903), the Science and Technology Commission of Shanghai Municipality (09PJ1404200, 09DJ1400400) and ECNU Graduate Student Fostering Project to Qinqin Gao.

\section{References}

1 Law JA, Jacobsen SE. Establishing, maintaining and modifying DNA methylation patterns in plants and animals. Nat Rev Genet 2010; 11:204-220.

2 Taberlay PC, Jones PA. DNA methylation and cancer. Prog Drug Res 2011; 67:1-23.
3 Chen T, Li E. Structure and function of eukaryotic DNA methyltransferases. Curr Top Dev Bio 2004; 60:55-89.

4 Leonhardt H, Page AW, Weier HU, Bestor TH. A targeting sequence directs DNA methyltransferase to sites of DNA replication in mammalian nuclei. Cell 1992; 71:865-873.

5 Liu Y, Oakeley EJ, Sun L, Jost JP. Multiple domains are involved in the targeting of the mouse DNA methyltransferase to the DNA replication foci. Nucleic Acids Res 1998; 26:1038-1045.

6 Chuang LS, Ian HI, Koh TW, et al. Human DNA-(cytosine-5) methyltransferase-PCNA complex as a target for p21WAF1. Science 1997; 277:1996-2000.

7 Bostick M, Kim JK, Esteve PO, et al. UHRF1 plays a role in maintaining DNA methylation in mammalian cells. Science 2007; 317:1760-1764.

8 Sharif J, Muto M, Takebayashi S, et al. The SRA protein Np95 mediates epigenetic inheritance by recruiting DNMT1 to methylated DNA. Nature 2007; 450:908-912.

9 Woo HR, Pontes O, Pikaard CS, Richards EJ. VIM1, a methylcytosine-binding protein required for centromeric heterochromatinization. Genes Dev 2007; 21:267-277.

10 Feng S, Cokus SJ, Zhang X, et al. Conservation and divergence of methylation patterning in plants and animals. Proc Natl Acad Sci USA 2010; 107:8689-8694.

11 Fujimori A, Matsuda Y, Takemoto Y, et al. Cloning and mapping of Np95 gene which encodes a novel nuclear protein associated with cell proliferation. Mamm Genome 1998; 9:10321035.

12 Hopfner R, Mousli M, Jeltsch JM, et al. ICBP90, a novel human CCAAT binding protein, involved in the regulation of topoisomerase IIalpha expression. Cancer Res 2000; 60:121128.

13 Unoki M, Nishidate T, Nakamura Y. ICBP90, an E2F-1 target, recruits $\mathrm{HDAC} 1$ and binds to methyl-CpG through its SRA domain. Oncogene 2004; 23:7601-7610.

14 Arita K, Ariyoshi M, Tochio H, Nakamura Y, Shirakawa M. Recognition of hemi-methylated DNA by the SRA protein UHRF1 by a base-flipping mechanism. Nature 2008; 455:818821.

15 Avvakumov GV, Walker JR, Xue S, et al. Structural basis for recognition of hemi-methylated DNA by the SRA domain of human UHRF1. Nature 2008; 455:822-825.

16 Hashimoto H, Horton JR, Zhang X, et al. The SRA domain of UHRF1 flips 5-methylcytosine out of the DNA helix. Nature 2008; 455:826-829.

17 Karagianni P, Amazit L, Qin J, Wong J. ICBP90, a novel methyl K9 $\mathrm{H} 3$ binding protein linking protein ubiquitination with heterochromatin formation. Mol Cell Biol 2008; 28:705717.

18 Nady N, Lemak A, Walker JR, et al. Recognition of multivalent histone states associated with heterochromatin by UHRF1 protein. J Biol Chem 286:24300-24311.

19 Rottach A, Frauer C, Pichler G, et al. The multi-domain protein Np95 connects DNA methylation and histone modification. Nucleic Acids Res 2010; 38:1796-1804.

20 Mori T, Li Y, Hata H, Kochi H. NIRF is a ubiquitin ligase that is capable of ubiquitinating PCNP, a PEST-containing nuclear protein. FEBS Lett 2004; 557:209-214.

21 Mori T, Li Y, Hata H, Ono K, Kochi H. NIRF, a novel RING 
finger protein, is involved in cell-cycle regulation. Biochem Biophys Res Commun 2002; 296:530-536.

22 Mousli M, Hopfner R, Abbady AQ, et al. ICBP90 belongs to a new family of proteins with an expression that is deregulated in cancer cells. Br J Cancer 2003; 89:120-127.

23 Lachner M, O'Carroll D, Rea S, Mechtler K, Jenuwein T. Methylation of histone $\mathrm{H} 3$ lysine 9 creates a binding site for HP1 proteins. Nature 2001; 410:116-120.

24 Bannister AJ, Zegerman P, Partridge JF, et al. Selective recognition of methylated lysine 9 on histone H3 by the HP1 chromo domain. Nature 2001; 410:120-124.

25 Qiu J, Shi G, Jia Y, et al. The X-linked mental retardation gene PHF8 is a histone demethylase involved in neuronal differentiation. Cell Res 2010; 20:908-918.

26 Yu L, Wang Y, Huang S, et al. Structural insights into a novel histone demethylase PHF8. Cell Res 2010; 20:166-173.

27 Zegerman P, Canas B, Pappin D, Kouzarides T. Histone H3 lysine 4 methylation disrupts binding of nucleosome remodeling and deacetylase (NuRD) repressor complex. J Biol Chem 2002; 277:11621-11624.

28 Nishioka K, Chuikov S, Sarma K, et al. Set9, a novel histone H3 methyltransferase that facilitates transcription by precluding histone tail modifications required for heterochromatin formation. Genes Dev 2002; 16:479-489.

29 Wang C, Shen J, Yang Z, et al. Structural basis for site-specific reading of unmodified R2 of histone $\mathrm{H} 3$ tail by UHRF1 PHD finger. Cell Res 2011; 21:1379-1382.

$30 \mathrm{Hu}$ L, Li Z, Wang P, Lin Y, Xu Y. Crystal structure of PHD domain of UHRF1 and insights into recognition of unmodified histone H3 arginine residue 2. Cell Res 2011; 21:1374-
1378.

31 Rajakumara E, Wang Z, Ma H, et al. PHD Finger Recognition of Unmodified Histone H3R2 Links UHRF1 to Regulation of Euchromatic Gene Expression. Molecular cell 2011; 43:275284.

32 Papait R, Pistore C, Grazini U, et al. The PHD domain of Np95 (mUHRF1) is involved in large-scale reorganization of pericentromeric heterochromatin. Mol Biol Cell 2008; 19:3554-3563.

33 Uemura T, Kubo E, Kanari Y, et al. Temporal and spatial localization of novel nuclear protein NP95 in mitotic and meiotic cells. Cell Struct Funct 2000; 25:149-159.

34 Nye AC, Rajendran RR, Stenoien DL, et al. Alteration of large-scale chromatin structure by estrogen receptor. $\mathrm{Mol}$ Cell Biol 2002; 22:3437-3449.

35 Meilinger D, Fellinger K, Bultmann S, et al. Np95 interacts with de novo DNA methyltransferases, Dnmt3a and Dnmt3b, and mediates epigenetic silencing of the viral CMV promoter in embryonic stem cells. EMBO Rep 2009; 10:1259-1264.

36 Kim JK, Esteve PO, Jacobsen SE, Pradhan S. UHRF1 binds G9a and participates in p21 transcriptional regulation in mammalian cells. Nucleic Acids Res 2009; 37:493-505.

37 Pichler G, Wolf P, Schmidt CS, et al. Cooperative DNA and histone binding by UHRF2 links the two major repressive epigenetic pathways. $J$ Cell Biochem 2011; 112:2585-2593.

38 Dignam JD, Lebovitz RM, Roeder RG. Accurate transcription initiation by RNA polymerase II in a soluble extract from isolated mammalian nuclei. Nucleic Acids Res 1983; 11:14751489.

(Supplementary information is linked to the online version of the paper on the Cell Research website.) 